\title{
INFLUÊNCIA DA ADIÇÃO DE BORO NA ESTRUTURA DO FERRO FUNDIDO BRANCO ALTO CROMO COM MOLIBDÊNIO, NO ESTADO BRUTO DE FUSÃO E TRATADO TERMICAMENTE *
}

Loudiana Mosqueira Antônio ${ }^{1}$

Dimas de Andrade Pacheco ${ }^{2}$ Cláudio Gonçalves de Oliveira ${ }^{3}$ Laura de Lima e Silva ${ }^{4}$ Cristina Godoy ${ }^{5}$

Ivete Peixoto Pinheiro 6

\section{Resumo}

Os ferros fundidos brancos alto cromo (High Chromiun Cast Iron - $\mathrm{HCCl}$ ) são ligas ferrosas com alta resistência ao desgaste. Isso se deve à elevada fração volumétrica de carbonetos eutéticos (10 - 30\%) que é distribuída em uma matriz metálica constituída de austenita, martensita e carbonetos secundários. Nesse trabalho será estudado o efeito da adição de molibdênio e boro na estrutura dos $\mathrm{HCCl}$. Para alcançar tal objetivo, inicialmente foram fundidas ligas de $\mathrm{HCCl}$ da classe ASTM 532 IIIA com e sem adição de boro (e adição de molibdênio em 1\%). Posteriormente, as ligas foram tratadas termicamente passando por recozimento, têmpera (desestabilização) e revenimento. Após o tratamento térmico, as amostras foram analisadas por microscopia óptica e análise de difração de raios $X$ (DRX). Os resultados indicam microestruturas refinadas para amostras com molibdênio e sem boro (BFMo) e formação de carbonetos primários grandes em amostras com adição de molibdênio e boro (BFMoB). Além disso, o tratamento térmico provocou um refinamento da microestrutura da liga TTMo e na liga TTMoB foi observada pequena quantidade de austenita retida. Sendo assim, a adição de boro provocou a formação de carbonetos primários $\left(\mathrm{M}_{7} \mathrm{C}_{3}\right.$ - de maior tamanho) e secundários $\left(\mathrm{M}_{23} \mathrm{C}_{6}, \mathrm{M}_{3} \mathrm{C}\right)$ e promoveu a diminuição da quantidade de austenita retida.

Palavras-chave: Ferro fundido branco; Boro; Carbonetos; Microestrutura.

\section{INFLUENCE OF BORON ADDITION ON THE STRUCTURE OF HIGH CHROMIUM WHITE CAST IRON WITH MOLYBDENUM IN THE MELT STATE AND HEAT TREATED}

\section{Abstract}

High chromium cast iron $(\mathrm{HCCl})$ are ferrous alloys with high wear resistance. This is due to the high volumetric fraction of eutectic carbides $(10-30 \%)$ that is distributed in a metallic matrix consisting of austenite, martensite and secondary carbides. In this work the effect of the boron addition in the $\mathrm{HCCl}$ microstructure will be studied. To achieve this purpose, $\mathrm{HCCl}$ alloys of the ASTM 532 IIIA class were initially cast with and without boron addition (and molybdenum addition of $1 \%$ ). Subsequently, the alloys were thermally treated by annealing, quenching (austenite destabilization) and tempering. After the heat treatment, the samples were analyzed by optical microscopy and X-ray diffraction (XRD) analysis. The results showed fine microstructures for samples with molybdenum and without boron (BFMo) and formation of large primary carbides in samples with boron addition (BFMoB). In addition, the heat treatment resulted in a refinement of TTMo alloy microstructure and in the TTMoB alloy was observed small amount of retained austenite. Thus, the addition of boron caused the formation of primary $\left(\mathrm{M}_{7} \mathrm{C}_{3}\right)$ and secondary carbides $\left(\mathrm{M}_{23} \mathrm{C}_{6}, \mathrm{M}_{3} \mathrm{C}\right)$ and led to a decrease of the amount of retained austenite.

Keywords: White cast iron; Boron; Carbides, Microstructure. 
1 Engenheira metalurgista, mestranda, Departamento de Engenharia de Materiais, Centro Federal de Educação Tecnológica de Minas Gerais, Belo Horizonte, Minas Gerais - Brasil.

2 Engenheiro metalurgista, mestrando, Departamento de Engenharia Metalúrgica, Universidade Federal de Minas Gerais, Belo Horizonte, Minas Gerais.

3 Engenheiro mecânico, doutorando, Departamento de Engenharia Metalúrgica, Universidade Federal de Minas Gerais, Belo Horizonte, Minas Gerais.

4 Graduanda em Engenharia Metalúrgica, Departamento de Engenharia Metal Universidade Federal de Minas Gerais, Belo Horizonte, Minas Gerais.

5 Física, doutora, Departamento de Engenharia Metalúrgica, Universidade Federal de Minas Gerais, Belo Horizonte, Minas Gerais.

6 Engenheira metalurgista, doutora, Departamento de Engenharia de Materiais, Centro Federal de Educação Tecnológica de Minas Gerais, Belo Horizonte, Minas Gerais - Brasil.. 


\section{INTRODUÇÃO}

Os ferros fundidos brancos de alto cromo (High Chromiun Cast Iron - $\mathrm{HCCl}$ ) são ligas ferrosas com uma quantidade $11-30 \%$ de cromo e 1,8-3,6\% de carbono, podem apresentar alguns elementos de liga como silício, molibdênio, manganês, cobre e níquel. Sua microestrutura é resultante da composição química, da taxa de extração de calor durante a solidificação e da velocidade de resfriamento após a solidificação [1].

Esse material é utilizado em aplicações em que elevada resistência ao desgaste é necessária (equipamentos de moagem, carcaças de bombas, revestimentos de moinhos e outros itens sujeitos a desgaste). Isso se deve à elevada fração volumétrica de carbonetos eutéticos $\left(\mathrm{M}_{7} \mathrm{C}_{3}\right)$ (entre 10 e $\left.30 \%\right)$ que é distribuída em uma matriz metálica constituída de diferentes proporções de austenita, martensita e carbonetos secundários $\left(\mathrm{M}_{23} \mathrm{C}_{6}\right.$ e $\left.\mathrm{M}_{3} \mathrm{C}\right)$, dependendo do histórico térmico [1,2].

Para alcançar as propriedades desejadas dos ferros fundidos brancos alto cromo, é necessaria uma adequação da composição química, realizada de acordo com o efeito de cada elemento. Essa adequação se faz necessária, porque apesar de o teor de cromo ser bastante elevado (12 a 30\%), a maior parte está combinada com o carbono na forma de carbonetos. Dois dos elementos que podem ser adicionados são: molibdênio (Mo) e boro (B). O molibdênio é adicionado em teores entre 0,5 e $3,5 \%$ e atua para suprimir a formação de perlita e aumentar a temperabilidade inibindo de forma eficaz a precipitação de carboneto secundário durante o resfriamento [3]. O boro é adicionado ao $\mathrm{HCCl}$ com o objetivo de aumentar a resistência ao desgaste sem diminuir a tenacidade, formando boretos em vez de carbonetos. Uma pequena quantidade de boro pode aumentar o endurecimento dos aços [4].

Quando maior dureza e maior resistência ao desgaste são desejadas, o tratamento térmico de desestabilização é empregado. A desestabilização consiste no tratamento equivalente à têmpera nos aços, porém com o emprego de maior tempo de austenitização, visando desestabilizar a austenita. Especificamente, o tratamento térmico consiste em promover a precipitação de carbonetos secundários visando aumentar a temperatura de início da transformação martensítica $\left(\mathrm{M}_{\mathrm{s}}\right)$ e a temperatura de final da transformação martensítica $\left(\mathrm{M}_{\mathrm{f}}\right)$, diminuindo a quantidade de austenita retida (estabilizada) no material [1].

Nesse trabalho, foi realizada a fusão de ligas de ferro fundido alto cromo $(25 \%$ de cromo) com e sem adição de boro (0,5\%). Essas ligas foram tratadas termicamente (recozimento, desestabilização e revenimento) e posteriormente foi avaliado o efeito da adição do boro na microestrutura.

\section{MATERIAIS E MÉTODOS}

A composição química desejada para as ligas está apresentada na Tabela 1.

Tabela 1 - Composição química nominal

\begin{tabular}{cccccccccc}
\hline \multirow{2}{*}{ LIGA } & \multicolumn{10}{c}{ COMPOSIÇÃO QUÍMICA (\%) } \\
\cline { 2 - 10 } & $C$ & $C r$ & $M n$ & Si & $P$ & $S$ & $N i$ & Mo & $B$ \\
\hline$M o$ & 2,65 & 25 & 1 & 1 & $<1$ & $<1$ & 1 & 1 & - \\
\hline$M o B$ & 3 & 25 & 1 & 1 & $<1$ & $<1$ & 1 & 1 & 0,5 \\
\hline \multicolumn{1}{c}{ Fonte: Próprio autor }
\end{tabular}


As ligas foram fundidas em forno elétrico à indução de média frequência, marca Inductotherm e capacidade do cadinho de $50 \mathrm{Kg}$. A análise química das ligas após a fusão foi realizada em Espectrômetro de emissão óptica da marca Spectromax. Para a detecção do boro na liga, foi necessário realizar uma análise química com abertura da amostra, devido ao baixo número atômico desse elemento.

Foi realizado um tratamento térmico nas ligas de acordo com a norma ASTM A532 (2014). As ligas foram recozidas a uma temperatura de $730^{\circ} \mathrm{C}$ por $2,5 \mathrm{~h}$; depois austenitizadas a $1020^{\circ} \mathrm{C}$ por $2,5 \mathrm{~h}$ com resfriamento forçado ao ar e depois revenidas a $250^{\circ} \mathrm{C}$ por $2 \mathrm{~h}$. Foi utilizada uma rampa de aquecimento e resfriamento de $110^{\circ} \mathrm{C} / \mathrm{h}$. Nas amostras que tiveram o resfriamento forçado não foi utilizada uma rampa de aquecimento.

A análise metalográfica das ligas foi realizada embutindo as amostras a quente (baquelite), lixando em lixas de granulometria 80, 220, 400, 600 e 1200 mesh, respectivamente e polindo em panos com pasta de diamante de 9 , 3 e $1 \mu \mathrm{m}$, respectivamente. Posteriormente, as amostras foram atacadas com o reagente Vilela (1g de ácido pícrico $+5 \mathrm{~mL}$ de ácido clorídrico $+100 \mathrm{~mL}$ de álcool etílico P.A.).

Para identificar as fases presentes na microestrutura foi realizada a difração de raios $X$ no Laboratório de Cristalografia da UFMG.

\section{RESULTADOS E DISCUSSÃO}

A composição das ligas após a análise química está apresentada na Tabela 2. Comparando a Tabela $1 \mathrm{com}$ a Tabela 2, é possível observar que a fusão ocorreu de maneira eficiente, uma vez que as composições obtidas foram as pré-determinadas.

Tabela 2- Composição química das ligas obtidas experimentalmente

\begin{tabular}{cccccccccc}
\hline \multirow{2}{*}{ LIGA COMIÇA QUÍMICA (\%) } \\
\cline { 2 - 10 } & $C$ & $C r$ & $M n$ & Si & $P$ & $S$ & $N i$ & Mo & $B$ \\
\hline Mo & 2,92 & 24,6 & 0,791 & 1 & 0,0327 & 0,008 & 0,547 & 1 & - \\
\hline MoB & 3,01 & 24,75 & 1,15 & 0,847 & 0,0356 & 0,0101 & 0,406 & 0,966 & 0,7 \\
\hline \multicolumn{1}{c}{ Fonte: Próprio autor }
\end{tabular}

A microestrutura das amostras de $\mathrm{HCCl}$ com e sem adição de boro podem ser vistas nas Figuras 1 e 2. Na liga com Mo no estado bruto de fusão (BFMo) (Figura 1 a e c), é possível observar uma microestrutura muito fina, com formação de carbonetos primários do tipo $\mathrm{M}_{7} \mathrm{C}_{3}$. Esse refinamento pode estar relacionado à presença de molibdênio na composição da liga. Esse elemento, que comumente é adicionado às ligas ASTM A532 tipo IIIA, possui o efeito de refinamento da microestrutura. Esse resultado está de acordo com um estudo sobre o efeito da adição de molibdênio em $\mathrm{HCCl}$ [5]. Esses autores encontraram que com teores de $13 \%$ de molibdênio houve formação de carbonetos primários muito finos $\left(M_{7} C_{3}, M_{2} C\right.$ e MC) e em pequeno volume. Apesar do teor adicionado no presente trabalho ser de $1 \%$, o efeito de refino na microestrutura foi muito parecido com o encontrado para uma adição de $13 \%$ de molibdênio. É possível observar também a presença de austenita (partes brancas Figura 1c). Já na microestrutura da liga Mo tratada (TTMo) (Figura 1 b e d), é possível observar um refinamento da microestrutura, a presença de martensita, devido ao tratamento térmico e carbonetos primários. 
a
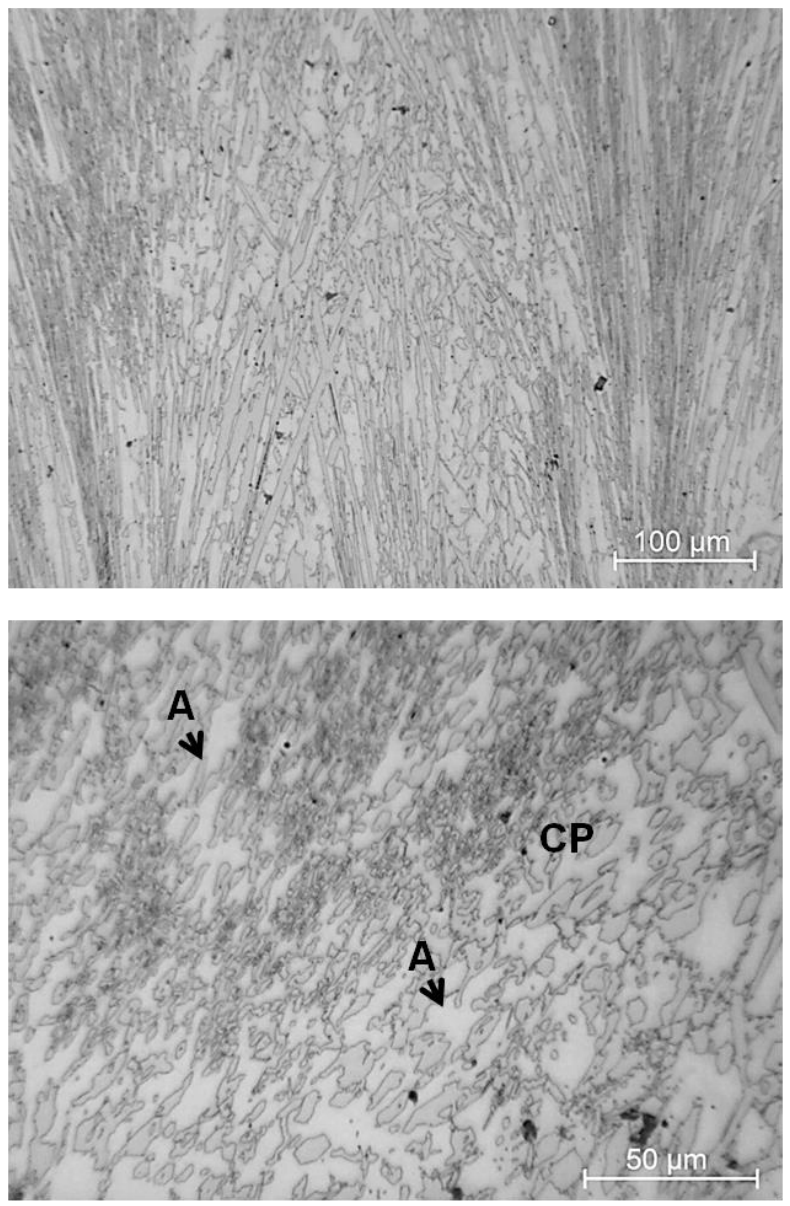

C b
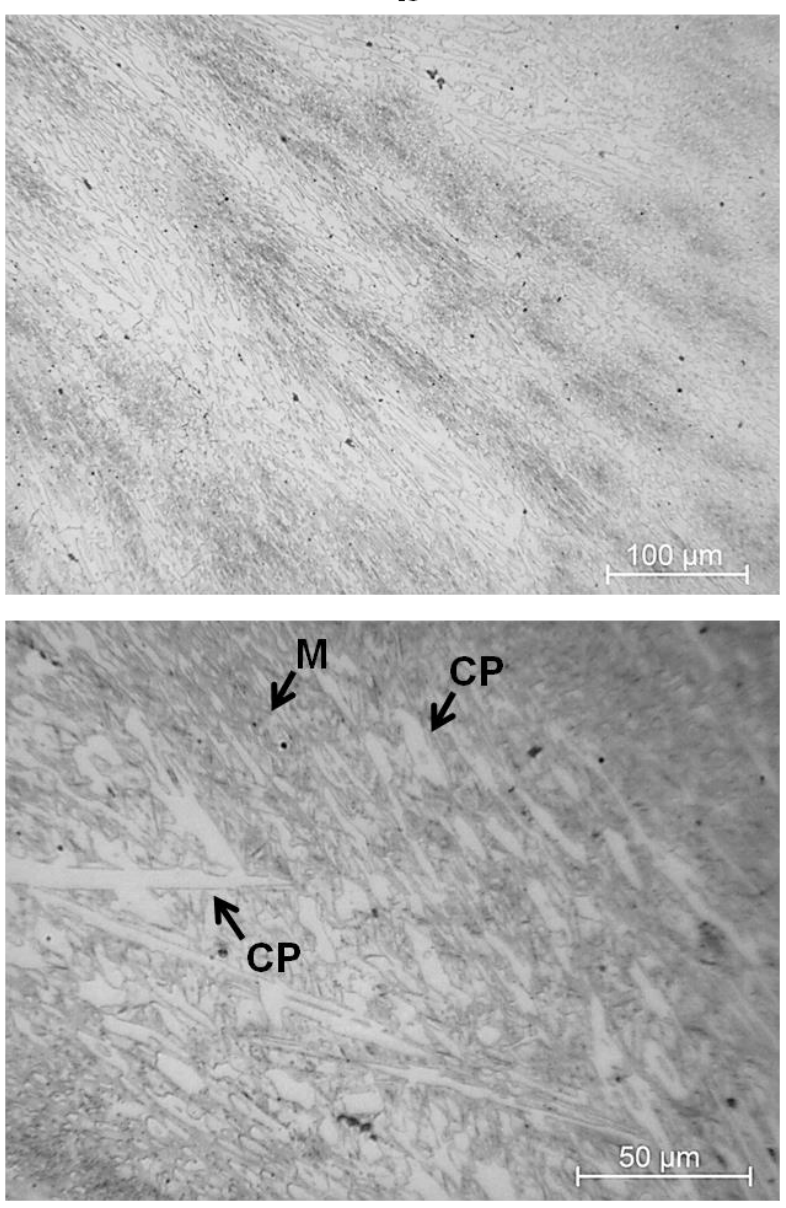

d

Figura 1 - Microscopia óptica da liga Mo

(a) BFMo (b) TTMo (c) BFMo (d) TTMo .

M: martensita; $\mathrm{A}$ : austenita; $\mathrm{CP}$ : carboneto primário.

Fonte: Próprio autor.

Na microestrutura da liga MoB no estado bruto de fusão (BFMoB) (Figura 2 a e c), é possível observar a presença de martensita, austenita e carbonetos primários grandes, indicados na Figura 2. A formação desses carbonetos está relacionada à adição de boro, pois ele substitui certo número de átomos de carbono. Os átomos de carbono substituídos pelo boro são transferidos dos carbonetos para o resto do metal fundido assegurando um aumento da saturação de carbono no metal, a formação de um número maior de núcleos de carbonetos e, finalmente, um aumento da fração de carbonetos na liga [6]. Além de haver grande formação de carbonetos, esperava-se que a microestrutura fosse mais fina, no entanto, isso não foi observado. Os carbonetos formados foram bem grandes. Isso pode ser explicado pelo teor de boro na liga. Um teor de boro de 0,1 a $0,3 \%$ em $\mathrm{HCCl}(27 \% \mathrm{Cr})$ provoca um refinamento dos carbonetos, porém a liga estudada possui um teor de aproximadamente $0,7 \%$ de boro e a observação de refino da literatura não foi verificada [7]. Na liga MoB tratada (TTMoB) (Figura 2 b e d), a microestrutura é um pouco mais refinada. A matriz é predominantemente martensítica, o que indica que 0 
tratamento de desestabilização da austenita, ou seja, transformação em martensita e precipitação de carbonetos secundários foi eficaz. Os carbonetos primários do tipo $\mathrm{M}_{7} \mathrm{C}_{3}$ continuam presentes na microestrutura da liga.

a
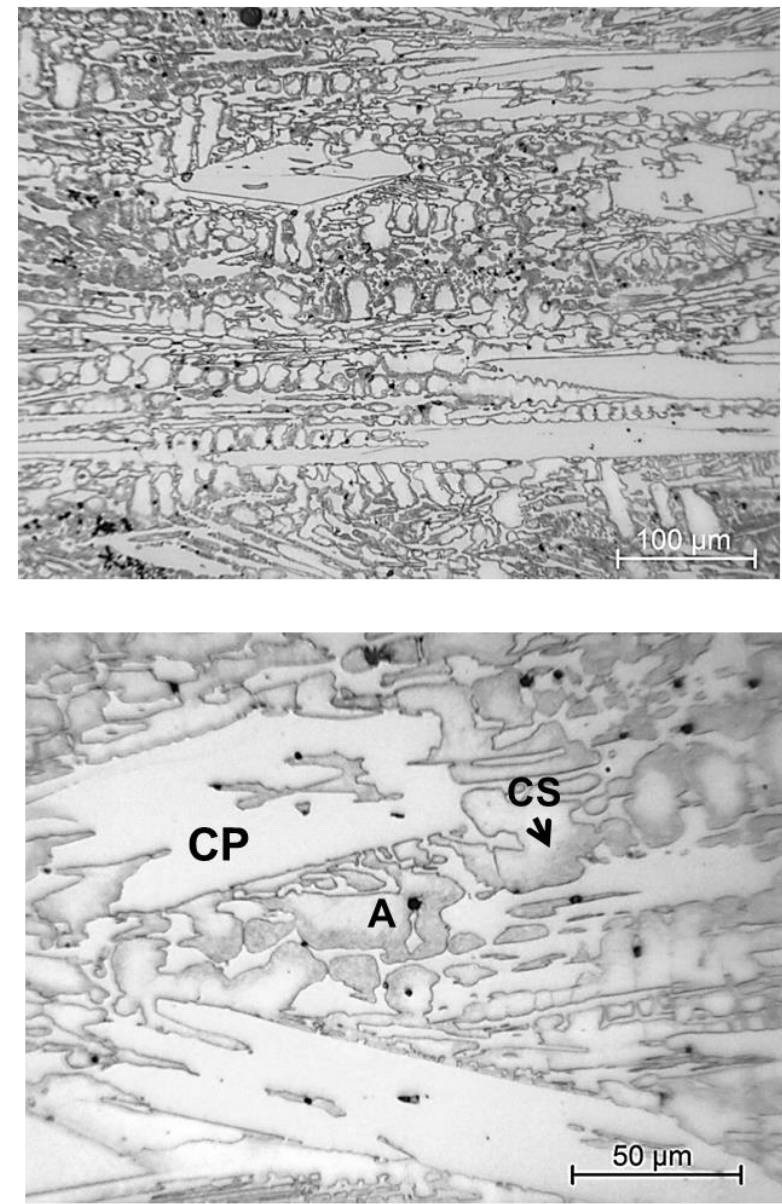

C
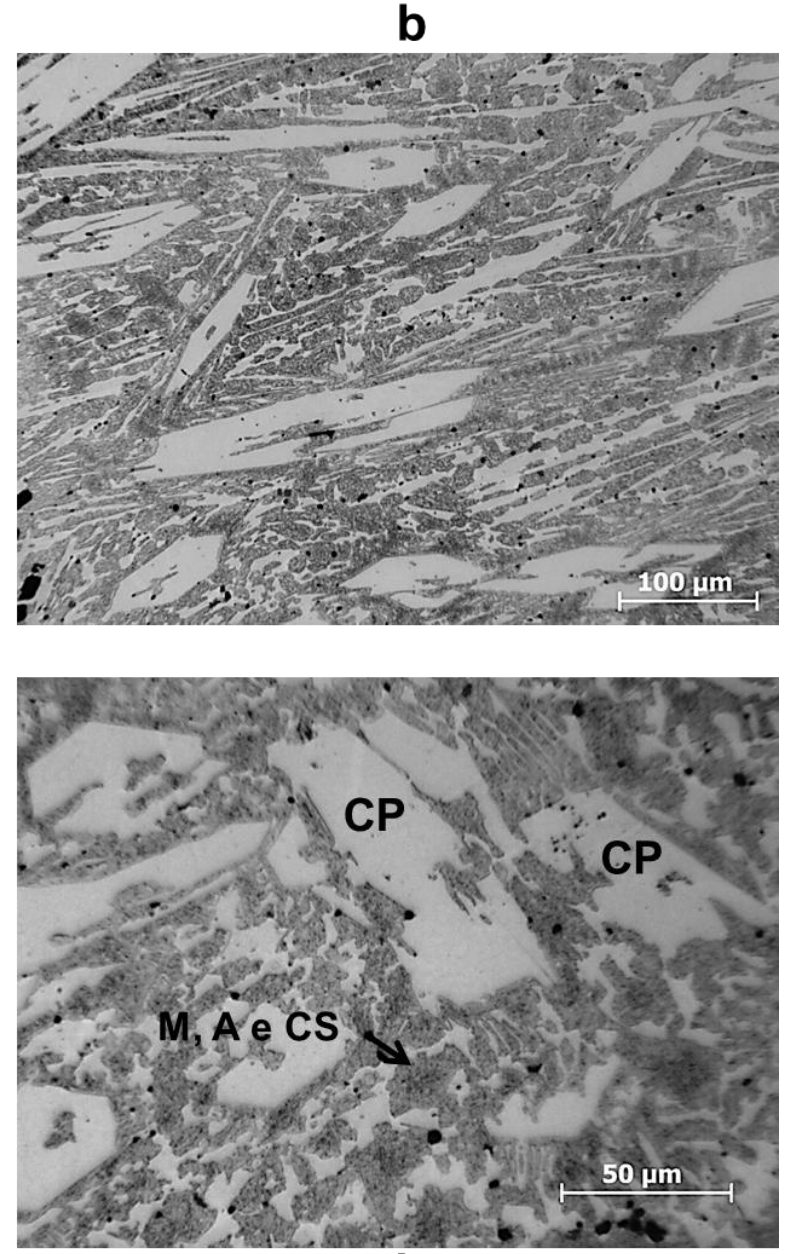

d

Figura 2 - Microscopia óptica da liga MoB

(a) BFMoB (b) TTMoB (c) BFMoB (d) TTMoB .

M: martensita; A: austenita; CP: carboneto primário; CS: Carboneto secundário. Fonte: Próprio autor.

Os difratogramas de raios $X$ das ligas BFMo, TTMo, BFMoB e TTMoB estão apresentados na Figura 3. Foi possível observar para a liga BFMo a presença de carbonetos primários $\mathrm{M}_{7} \mathrm{C}_{3}$ e uma matriz predominantemente austenítica com alguma ferrita (como mostrado na Figura 1 a e c). Na liga TTMo, há presença de carbonetos primários $\mathrm{M}_{7} \mathrm{C}_{3}$ em maior volume (maior intensidade dos picos característicos) e uma matriz, com martensita e austenita. Apesar do tratamento de desestabilização promover a transformação martensítica e a formação de carbonetos secundários diminuindo assim, a quantidade de austenita retida, foi observado no DRX um pico bem intenso de austenita, para a liga TTMo. Isso indica que a desestabilização da austenita não foi muito eficaz. Como no DRX da amostra TTMo não foi observada a presença de carbonetos secundários, a quantidade de austenita retida foi alta, pois a quantidade de carbono dissolvido na matriz é grande, diminuindo assim, a temperatura $\mathrm{M}_{\mathrm{s}}$. Os gráficos da Figura $5 \mathrm{a}$ e b mostram a relação entre o volume de carbonetos secundários (Figura 5a) e a quantidade de austenita retida (Figura 5b) com o tempo de desestabilização para um $\mathrm{HCCl}$ com $17 \%$ de 
cromo, $2 \%$ de molibdênio e $2,6 \%$ de carbono em um estudo de Bedolla-Jacuide, Arias e Hernández sobre a formação de carbonetos secundários [8]. É possível observar na Figura 5 que para uma maior fração volumétrica de carbonetos secundários, a quantidade de austenita retida diminui. Além disso, para temperaturas maiores de desestabilização, a quantidade de austenita retida na microestrutura aumenta, devido à maior quantidade de carbono dissolvido na austenita que faz com que a temperatura $\mathrm{M}_{\mathrm{s}}$ seja menor.

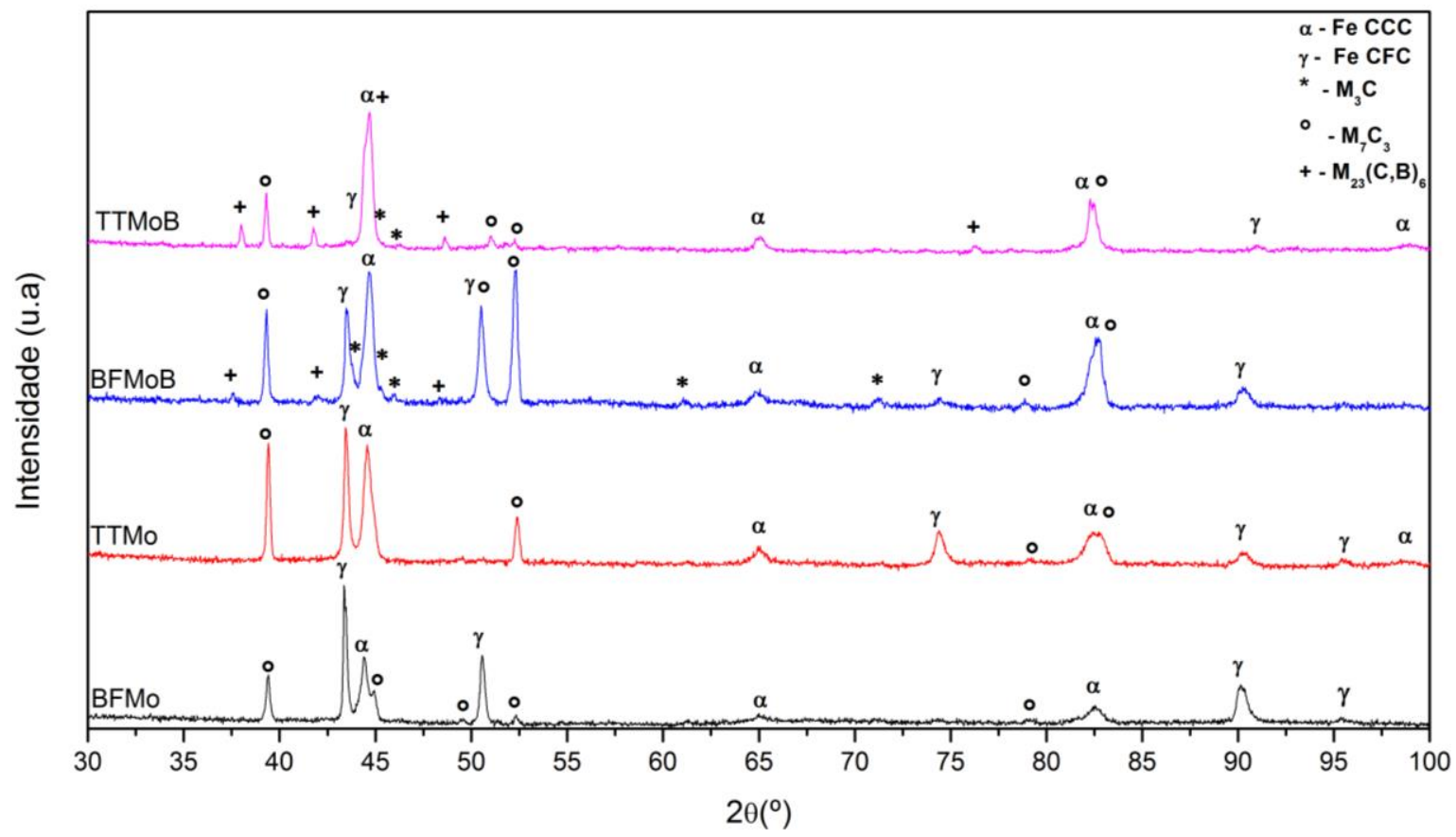

Figura 3 - DRX das ligas BFMo, TTMo, BFMoB e TTMoB Fonte: Próprio autor.

(a)

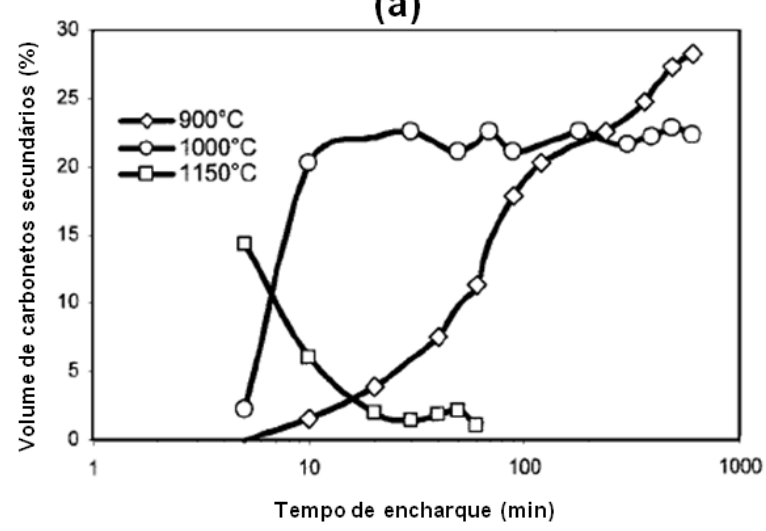

(b)

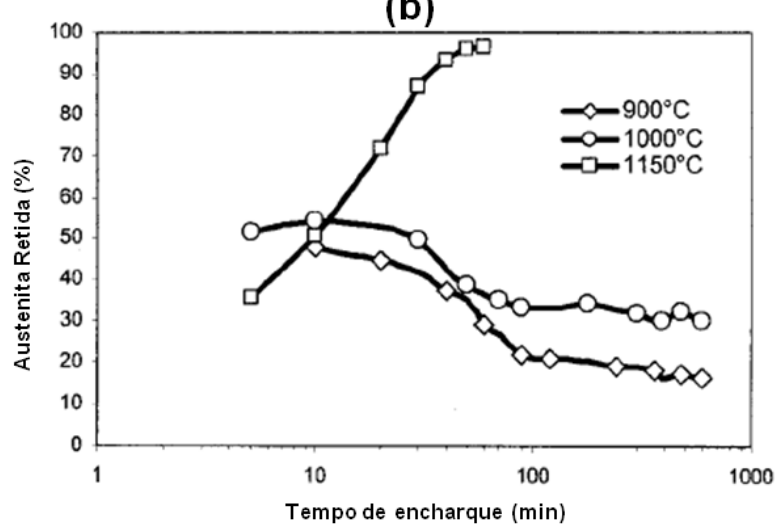

Figura 4 - Volume de carbonetos secundários e austenita retida como uma função do tempo de encharque da desestabilização

(a) Volume de carbonetos secundários (b) Austenita retida Fonte: Adaptado de [8].

$\mathrm{Na}$ liga $\mathrm{BFMoB}$, a quantidade de carbonetos primários é maior, uma vez que a intensidade dos picos característicos dessa fase é bem maior. A matriz é austenítica e ainda há presença de perlita ( $\mathrm{Fe}_{3} \mathrm{C}$-cementita e ferrita), o que é confirmado pelo DRX (presença da fase $\mathrm{M}_{3} \mathrm{C}$ ), e pela Figura 6 que indica a presença do constituinte perlita. Isso explica a grande intensidade de picos característicos do Fe CCC, que 
indicam tanto a presença de martensita quanto de ferrita. A formação de carbonetos secundários $\mathrm{M}_{23}(\mathrm{C}, \mathrm{B})_{6}$ também foi comprovada pelo $\mathrm{DRX}$, o que mostra que a adição de boro realmente propicia a formação dos mesmos. Na liga TTMoB, a matriz foi predominantemente martensítica com alguma austenita retida (figura 2 b e d). Pode-se observar que os picos característicos da estrutura CFC da austenita apresentam menor intensidade, indicando que o tratamento de desestabilização da austenita foi eficaz na formação de martensita. A precipitação de carbonetos secundários pode ser observada pelo difratograma também, o que explica a diminuição da quantidade de austenita retida. $O$ aumento da fração volumétrica de carbonetos precipitados ao longo do tempo resulta em redução do teor de carbono dissolvido na austenita e, portanto, aumento das temperaturas $M_{s}$ e $M_{f}$ [1]. A intensidade dos picos característicos do $\mathrm{M}_{23}(\mathrm{C}, \mathrm{B})_{6}$ aumenta da amostra BFMoB para TTMoB. Isso também é consequência do tratamento de desestabilização da austenita.

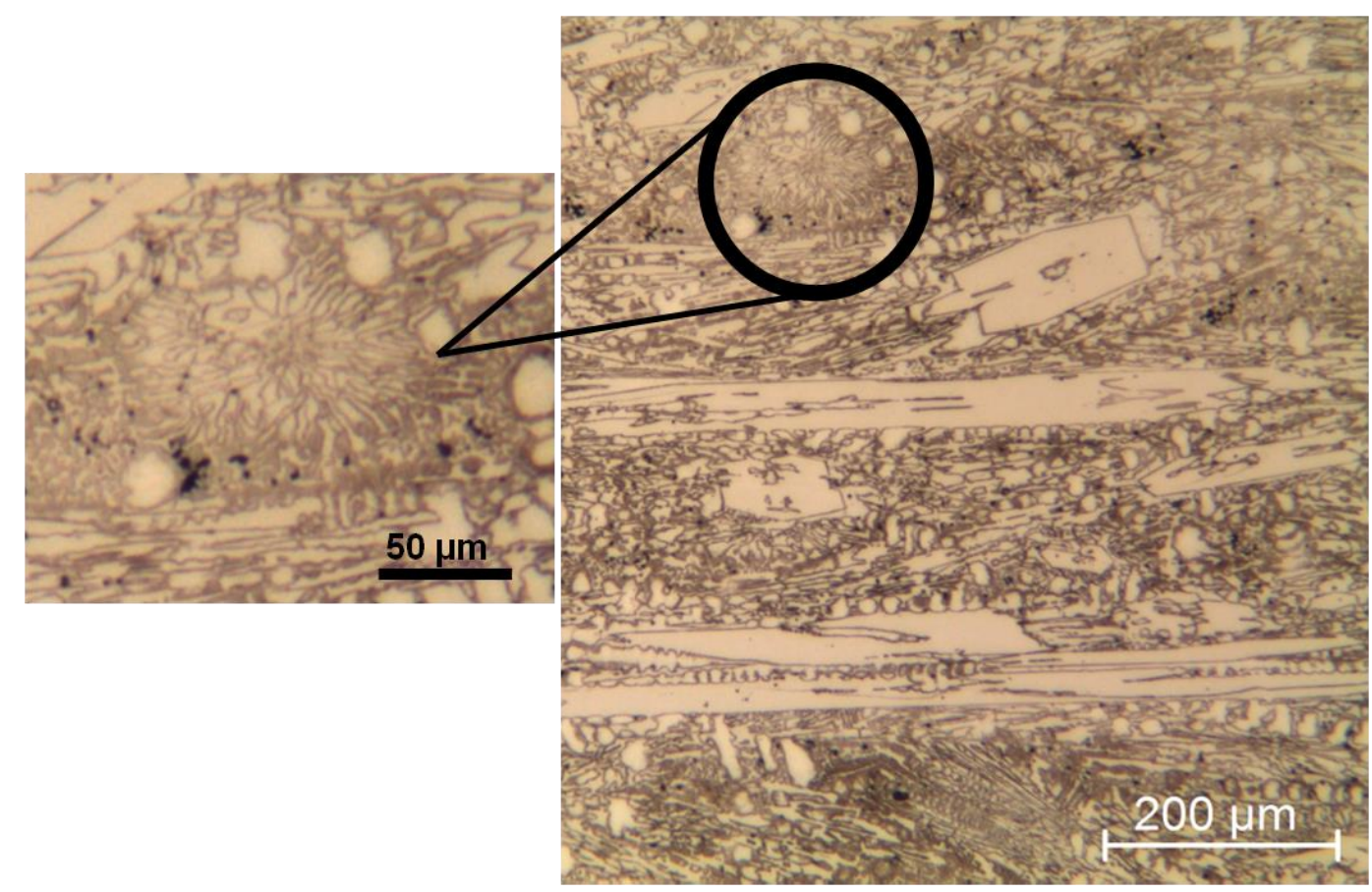

Figura 5 - Detalhe mostrando a perlita da liga BFMoB Fonte: Próprio autor.

\section{CONCLUSÃO}

A análise dos resultados nos permite chegar às seguintes conclusões:

- A presença de molibdênio no $\mathrm{HCCl}$ na liga BFMo, sem boro, leva a uma superestabilização da austenita (diminuindo a transformação de austenita para martensita) e leva também a um refinamento da microestrutura.

- A adição de boro na liga num teor de $0,7 \%$ (BFMoB) favorece a formação de carbonetos primários $\mathrm{M}_{7} \mathrm{C}_{3}$ grandes e de carbonetos secundários $\mathrm{M}_{23}(\mathrm{C}, \mathrm{B})_{6}$.

- O tratamento térmico de desestabilização (transformação da austenita em martensita) na liga com adição de molibdênio e boro (TTMoB) diminui a quantidade de austenita retida na microestrutura e aumenta a precipitação de carbonetos secundários $\mathrm{M}_{23}(\mathrm{C}, \mathrm{B})_{6}$ na microestrutura. 


\section{Agradecimentos}

CAPES, CEFET-MG, Laboratório de Cristalografia da UFMG

\section{REFERÊNCIAS}

1 Ortega $\mathrm{P}$, Bernardini $\mathrm{P}$, Torres $\mathrm{L}$. Otimização do tratamento térmico de desestabilização do ferro fundido branco de alto cromo classe II d usado em moinhos rolo sobre pista de usinas termoelétricas. Citenel. Florianópolis; 2005.

2 Kasama A. Resistência ao desgaste de ferro fundido branco alto cromo conformado por spray. In: CBECiMat. Anais do 15․ 2002. p.3789-3794.

3 Tabrett C, Sare I, Ghomashchi M. Microstructure-property relationships in high chromium white iron alloys. International Materials Reviews. 1996;41(2):59-82.

4 Zeytin H, Yildirim H, Berme B, Duduoĝlu S, Kazdal G, Deniz A. Effect of boron and heat treatment on mechanical properties of white cast iron for mining application. Journal of Iron and Steel Research, International. 2011;18(11):3139.

5 Chung R, Tang X, Li D, Hinckley B, Dolman K. Microstructure refinement of hypereutectic high $\mathrm{Cr}$ cast irons using hard carbide-forming elements for improved wear resistance. Wear. 2013;301(1-2):695-706.

6 Tomovic-Petrovic S, Markovic S, Zec S. The effect of boron on the amount and type of carbides in chromium white irons. Journal of the Serbian Chemical Society. 2002;67(10):697-707.

7 Jacuinde A, Mejía I, Verduzco J. Abrasive wear of boron-added highchromium white irons. In: 4th International Conference on Tribology in Manufacturing Processes. Nice; 2010.

8 Bedolla-Jacuide A, Arias L, Hernández B. Kinetics of secondary carbides precipitation in a high-chromium white iron. Journal of Materials Engineering and Performance. 2003;12(4):371-382. 